\title{
Diet quality of Singaporean children aged 6 to 12 years old
}

\author{
L. Santos-Merx ${ }^{1}$, J. Low ${ }^{1}$, N. Duriraju ${ }^{1}$, M. Chu ${ }^{1}$, J. Ong ${ }^{1}$, S. Hopkins ${ }^{2}$, C.J. Seal ${ }^{3}$ and \\ I.A. Brownlee \\ ${ }^{1}$ Food and Human Nutrition, Newcastle University in Singapore, Singapore, ${ }^{2}$ Cereal Partners Worldwide, Lausanne, \\ Switzerland, ${ }^{3}$ Human Nutrition Research Centre, Institute of Cellular Medicine, Newcastle University, Newcastle \\ upon Tyne, UK and ${ }^{4}$ CSIRO Health and Biosecurity, Adelaide, Australia.
}

In Singapore, healthy eating guidelines have been designed to improve food choices and create healthy habits for life, especially among young people. Nonetheless, limited data exist on dietary habits of Singaporeans below 18 years of age ${ }^{(1,2)}$. The aim of the current study is to evaluate the diet quality of Singaporean children aged 6 to 12 years.

Dietary intake from two 24-hour food recalls of 559 children, previously collected for another study ${ }^{(2)}$ were evaluated using a dietary index based on the Health Promotion Board food-based dietary guidelines ${ }^{(3)}$. Differences regarding frequencies on dietary index components and total score were analysed using Chi-square test, $p<0.05$.

Overall, $62 \%$ of children had excessive intake of sodium and $44 \%$ consumed deep fried food. At least $40 \%$ of children ingested sugary products (confectionary, drinks and sweetened dairy) above guideline frequencies. Moreover, wholegrains, fruits and vegetables were rarely consumed. When contrasting data across tertiles of the dietary index score, and as expected, there were more high scorers who met requirements for healthy items than mid and low scorers $(p<0 \cdot 05$, Figure 1$)$. Moreover, there were fewer high scorers who ingested sweetened dairy products, sugary items, and deep fried food than mid and low scorers. Nonetheless, at least $20 \%$ of high scorers consumed one or more of these less healthy items. Additionally, about half of high scorers ingested foods rich in sodium above the maximum recommended guideline. Whole grains were hardly consumed across the 3 dietary categories (Figure 1).

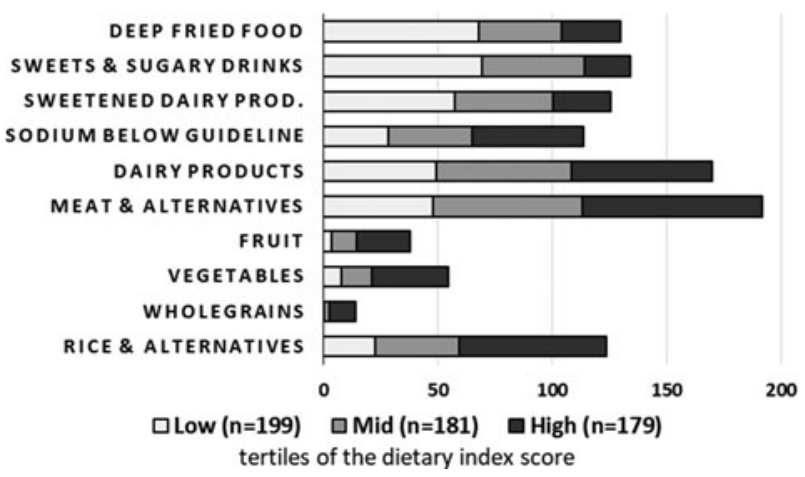

Figure 1. Percentage of low, mid and high scorers meeting dietary components.

More boys than girls consumed meat and deep fried food $(p<0.05)$. No other differences were found between genders. Dietary Index Score and age shared a small negative but significant relationship $(\rho=-0 \cdot 148 ; p=0.0001)$. Although weak, this value suggests as age increases the diet quality for these Singaporean children gets slightly worse.

Despite some children showed high adherence to healthy eating guidelines, current results suggest public health strategies in children should focus in decreasing the intake of sugary, salty and deep fried food items alongside existing efforts in increasing fruit, vegetables and wholegrains intake.

1. Ang KL \& Foo S (2002) Health Education 102, 239-248.

2. Neo JE, et al. (2016) J Nutr Sci 5, e33.

3. Health Promotion Board (2012) Birth to eighteen years - Dietary tips for your child's wellbeing. 